\title{
Generation of narrowband, high-intensity, carrier-envelope phase-stable pulses tunable between 4 and $18 \mathrm{THz}$
}

\author{
Biaolong Liu ${ }^{1}$, Michael Först ${ }^{1,}$, Hubertus Bromberger ${ }^{1}$, Andrea Cartella ${ }^{1}$, Thomas Gebert ${ }^{1}$, \\ and Andrea Cavalleri ${ }^{1,2}$ \\ ${ }^{1}$ Max Planck Institute for the Structure and Dynamics of Matter, Hamburg, Germany \\ ${ }^{2}$ Department of Physics, Oxford University, Clarendon Laboratory, Parks Road, Oxford, United \\ Kingdom
}

\begin{abstract}
We demonstrate the generation of narrowband $(<1 \mathrm{THz})$ highenergy $(\sim 2 \mu \mathrm{J})$ carrier-envelope phase-stable pulses, tunable between 4 and $18 \mathrm{THz}$ as achieved by difference-frequency mixing between chirped nearinfrared pulses in organic DSTMS.
\end{abstract}

\section{Introduction}

Many applications in femtosecond optical science require that one is able to generate highenergy pulses in the range between 1 and $20 \mathrm{THz}$. For example, one of the most interesting areas of condensed matter research in the past few years has been the use of $\mathrm{THz}$ light to control lattice vibrations [1] and with that the functionality of many correlated electron materials like, e.g., high-temperature superconductors [2]. However, the lack of powerful sources with frequencies $<15 \mathrm{THz}$ has restricted this selective control to a particular set of higher-energy vibrational modes in these solids.

The availability of large organic crystals, which exhibit significant nonlinear susceptibility and low absorption in the THz range, has opened up new possibilities [3]. Here, we present the generation of far-infrared narrowband carrier-envelope phase-stable pulses achieved by different frequency generation (DFG) between two chirped nearinfrared pulses in a DSTMS organic crystal. Sub-picosecond pulses tunable between 4 and $18 \mathrm{THz}$ are demonstrated, with a bandwidth of less than $1 \mathrm{THz}$ (FWHM) and pulse energies up to $\sim 2 \mu \mathrm{J}$.

\section{Experimental setup and results}

Figure 1(a) schematically displays the optical setup. A commercial Ti:sapphire amplifier, delivering $800-\mathrm{nm}$ wavelength pulses of $3 \mathrm{~mJ}$ energy and $\sim 100 \mathrm{fs}$ duration at $1 \mathrm{kHz}$ repetition rate was used to pump two identical three-stage optical parametric amplifiers (OPA). The OPAs were seeded by the same white light continuum to generate near-infrared

*Corresponding author: michael.foerst@mpsd.mpg.de 
pulses with correlated phase fluctuations, which could then be subtracted in the DFG process to result in a passively stable carrier-envelope phase of the $\mathrm{THz}$ radiation [4]. Each OPA generated signal output pulses of maximum $\sim 380 \mu \mathrm{J}$ energy and $\sim 130$ fs duration, which were tuned between 1.35 and $1.5 \mu \mathrm{m}$. The near-IR beams were collimated to diameters of $\sim 2 \mathrm{~mm}$, keeping the fluence incident on the DSTMS crystal below its damage threshold of $150 \mathrm{GW} / \mathrm{cm}^{2}$. Efficient DFG in the nonlinear DSTMS crystal requires type-0 phase matching, facilitated by polarizing both near-IR beams along the crystal a-axis and choosing a quasi-collinear geometry.
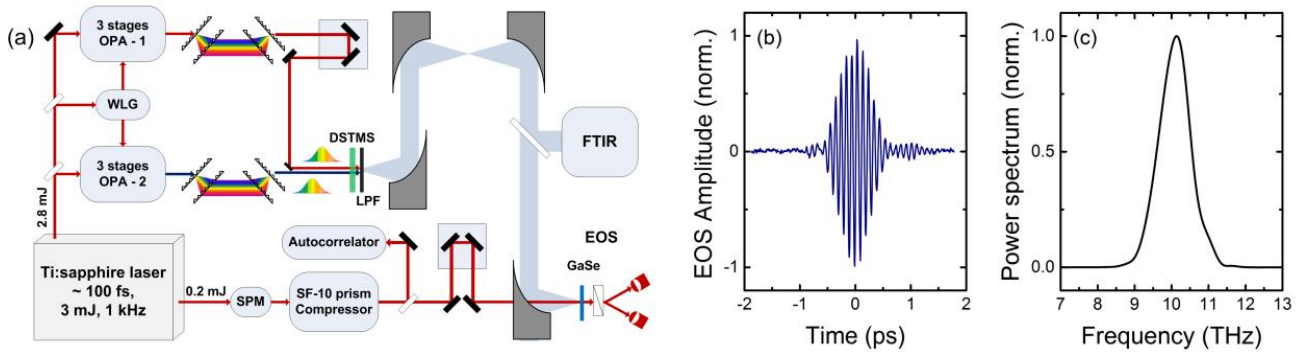

Fig.1. (a) Narrowband THz setup. Two separate OPAs, pumped by a Ti:sapphire laser, provide tunable signals which are linearly chirped afterwards for the DFG process in the DSTMS. The generated THz electric fields are characterized via FTIR or via electro-optic sampling in a GaSe (b) THz transient electric field generated by DFG in a $480 \mu \mathrm{m}$ thick DSTMS crystal as detected via electro-optic sampling in a GaSe crystal of $100 \mu \mathrm{m}$ thickness. (c) Corresponding power spectrum.

Equivalent amounts of negative linear chirp were introduced to the near-IR pulses by two stretchers built from high-efficiency transmission gratings. Consequently, two frequency combs with a single beating frequency propagate through the nonlinear DSTMS crystal to produce the THz electric fields. Stretching to about $600 \mathrm{fs}$ was chosen to suppress broadband $\mathrm{THz}$ emission from optical rectification whilst retaining sufficient field strength for efficient difference-frequency generation. The generated far-infrared pulses were transmitted through three $20-\mathrm{THz}$ low-pass filters to block the residual near-infrared beams.

Figure 1(b) shows a CEP-stable THz electric field generated in a $480 \mu \mathrm{m}$ thick DSTMS crystal by mixing two OPA signal wavelengths tuned to 1430 and $1500 \mathrm{~nm}$, respectively. This pulse, with a duration of $\sim 420 \mathrm{fs}$ (FWHM), was measured by electro-optic sampled in $100-\mu \mathrm{m}$ thick GaSe using $\sim 50$ fs gate pulses at $800 \mathrm{~nm}$ wavelength, as produced by spectral broadening and compression of the fundamental Ti:sapphire pulses. The corresponding power spectrum, plotted in Figure 1(c), is centered at $10 \mathrm{THz}$ with less than $1 \mathrm{THz}$ bandwidth. The long-term stability of the phase of the electric field was measured by consecutively sampling the $\mathrm{THz}$ pulses. Within four hours, the phase accumulated a linear drift of 0.14 with residual fluctuations of $0.03 \quad$ (standard deviation), likely due to thermal and mechanical instabilities in the optical setup.

The pulse energy of the electric field transient shown above, measured by a commercial thermopile detector, was $1.9 \mu \mathrm{J}$. Two parabolic mirrors were used to first collimate and then focus the $\mathrm{THz}$ beam onto the electro-optic detection crystal. A spot size of $180 \mu \mathrm{m}$ was determined from knife-edge measurements, resulting in a peak electric field of 3.7 $\mathrm{MV} / \mathrm{cm}$. The corresponding peak intensity was $17.8 \mathrm{GW} / \mathrm{cm} 2$.

Figure 2(a) shows Fourier transform interferometry (FTIR) traces of various electric field transients tuned across a broad spectral range below $20 \mathrm{THz}$. The corresponding Fourier transforms, shown in Fig. 2(b), confirm the tunability of the developed source between about 4 and $18 \mathrm{THz}$. To achieve maximum pulse energy at a desired $\mathrm{THz}$ frequency the center wavelengths of the two near-infrared pulses were tuned simultaneously. The amount of chirp on the two near-IR pulses was kept constant 
throughout these measurements, resulting in spectral bandwidths always below $1 \mathrm{THz}$. The pulse energy for these different frequencies varies between 1.3 and $1.9 \mu \mathrm{J}$, likely due to the frequency-dependent absorption coefficient of the DSTMS crystal.
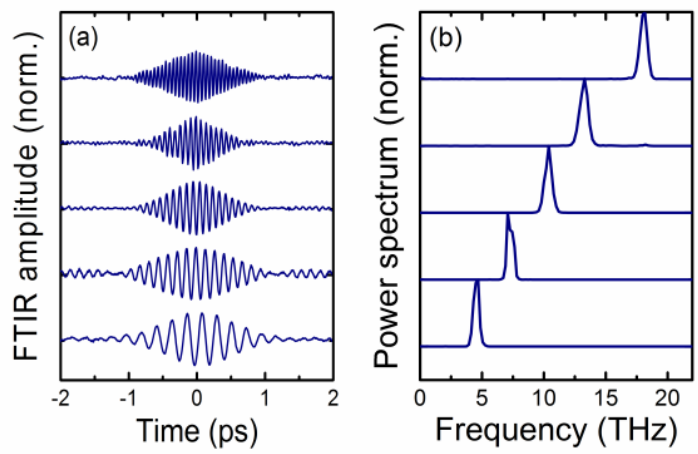

Fig.2. Normalized (a) FTIR interferograms and (b) corresponding Fourier transformations of THz transient electric fields, tuned by changing the difference frequency between the two linearly-chirped near-IR pulses.

\section{Conclusions}

In summary, we reported a table-top source of narrowband, high-intensity, carrier-envelope phase-stable far-infrared electromagnetic fields, tunable between 4 and $18 \mathrm{THz}$. Pulse energies of up to $\sim 2 \mu \mathrm{J}$ with peak electric field of $>3.5 \mathrm{MV} / \mathrm{cm}$ were demonstrated when pumping the system with 3-mJ pulses from a Ti:sapphire regenerative amplifier. Chirping the two near-IR pulses used in the THz generating difference frequency mixing process is crucial to suppress broadband $\mathrm{THz}$ radiation otherwise generated via optical rectification.

\section{References}

1. M. Först, C. Manzoni, S. Kaiser, Y. Tomioka, Y. Tokura, R. Merlin, and A. Cavalleri, Nature Physics 7, 854 (2011)

2. R. Mankowsky, M. Först and A. Cavalleri, Reports on Progress in Physics, 79, 6 (2016)

3. C. Vicario, B. Monoszlai, and C. P. Hauri, Phys. Rev. Lett. 112, 213901, (2014)

4. C. Manzoni, G. Cerullo and S. De Silvestri, Opt. Lett. 29, 2668 (2004). 